\title{
New Experimental Results on the Casimir Effect
}

\author{
V. M. Mostepanenko \\ Departamento de Física, Universidade Federal da Paraíba, João Pessoa, \\ 58059-970, Brazil \\ (on leave from A.Friedmann Laboratory for Theoretical Physics, St.Petersburg, Russia) \\ E-mail: mostep@fisica.ufpb.br
}

Received 7 January, 2000

\begin{abstract}
New experiments are discussed on measuring the Casimir force between metallic surfaces. One of them uses torsion pendulum and the other one - atomic force microscope. The claimed agreement of experimental data with a theory is analyzed. A $5 \%$ level of agreement obtained with a torsion pendulum is shown to be in contradiction with the values of surface roughness, finite conductivity and temperature corrections to the Casimir force. A 1\% agreement (at the closest separations) obtained with an atomic force microscope is confirmed by the use of more exact theory taking into account higher order surface roughness and conductivity corrections.
\end{abstract}

\section{Introduction}

Casimir effect arises in bounded domains and in spaces with non-trivial topologies as a result of the distortion of the zero point vacuum fluctuation spectrum of quantized fields [1, 2]. It appears in the form of attractive or repulsive forces between macroscopic material boundaries in a vacuum.

An important feature of the Casimir effect is that even though it is quantum in nature, it predicts a force between macroscopic bodies. For two plane-parallel metallic plates of area $S=1 \mathrm{~cm}^{2}$ separated by a large distance (on the atomic scale) of $a=0.5 \mu \mathrm{m}$ the value of the attractive force acting between them is:

$$
F_{C}^{(0)}(a)=-\frac{\pi^{2}}{240} \frac{S \hbar c}{a^{4}}=2 \times 10^{-6} \mathrm{~N}
$$

As is seen from (1.1) the Casimir force depends only on the fundamental constants $\hbar$ and $c$, unlike other vacuum effects which depend on the charge, mass and coupling constants.

There exist only a few other macroscopic manifestations of quantum phenomena. Among them there are the famous ones such as Superconductivity, Superfluidity and the Quantum Hall effect. In the above macroscopic quantum effects the coherent behavior of large number of quantum particles plays an important role. Similarly the Casimir effect can be considered also as a macroscopic quantum effect as it takes place between boundaries placed at such large distances that the virtual photon emitted by an atom of one body cannot reach the second body during its lifetime. Nevertheless, the correllator of the quantized electromagnetic field in a vacuum state at all pairs of points belonging to the symmetrical opposite layers of different boundaries is not equal to zero. Hence correlated oscillations arise of the dipole moments of atoms situated at those points resulting in appearance of the Casimir force. (The opposite limit of attractive forces between closely spaced bodies is referred to as the van der Waals force). The clearest implication of the above is that greater attention traditionally given to the macroscopic quantum effects will also be received by the Casimir effect.

The Casimir effect plays an important role in a variety of fields of physics such as Quantum Field Theory, Gravitation and Cosmology, Atomic and Molecular Physics and Mathematical Physics.

In Quantum Field Theory the Casimir effect finds three main applications. In the bag model of Quantum Chromodynamics the Casimir energy of quark and gluon fields contributes up to $10 \%$ of the total nucleon energy [2-4]. In Kaluza-Klein field theories Casimir effect offers one of the most effective mechanisms for spontaneous compactification of extra spatial dimensions [5]. Moreover measurements of the Casimir force provide opportunities to obtain more strong constraints for the parameters of long-range interactions and light elementary particles predicted by the unified gauge theories of fundamental interactions [6-8].

In Gravitation and Cosmology the Casimir effect arises due to the non-trivial topology of space-time [9]. The vacuum polarization resulting from the Casimir effect can drive the inflation process in the cosmological models with non-Euclidean topology [10]. In the theory of structure formation of the Universe due to topological defects, the Casimir vacuum polarization near cosmic strings plays an important role [11].

In Condensed Matter Physics, the Casimir effect 
leads to attractive and repulsive forces between the material boundaries which depend on the geometry of the boundaries, on temperature, and the electrical and mechanical properties of the boundary surface [2,12-14]. It is responsible for some properties in thin films [15].

In Atomic Physics, the Casimir effect leads to the corrections to the energy levels of Rydberg states [16]. A number of the Casimir-type effects arise in Cavity Quantum Electrodynamics when the radiative processes and associated energy shifts are modified by the presence of cavity walls [17].

In Mathematical Physics the investigation of the Casimir effect has stimulated the development of powerful techniques like Riemann and Epstein zeta-function regularization and also by means of heat-kernel expansion $[18]$.

In the five decades following Casimir's discovery the field has been dominated by enormous theoretical output. Given the small forces involved experimental progress has been painfully limited. Before 1997 there have been two experimental attempts. The first by Sparnaay based on a spring balance [19] qualitatively showed the attractive force but was limited due to the $100 \%$ experimental error. In [20] the Casimir force between the Chromium covered plate and a spherical lens was measured also using a spring balance. No estimate for the accuracy was presented in [20]. Other measurements of the Casimir force between 1956-1996 were performed with the dielectric test bodies (see for e.g. [2]), which limits the accuracy due to strong dependence of the force on the dielectric properties.

Here we discuss the new experiments on the measuring of the Casimir force between metals. In Sec. II the experiment [21] is briefly discussed. In Sec. III the results of the experiment [22] are presented. Sec. IV contains the critical analysis of the relation between the experimental data of $[21,22]$ and theoretical approaches used in these papers. More complete theory which is in agreement with the results of [22] is the subject of Sec. V. Sec. VI contains conclusions and discussion.

\section{The experiment by S.K. Lam- oreaux}

The modern stage of the Casimir force measurements between metals was opened by the paper [21]. In this paper a torsion pendulum was used to measure the force between $C u$ plus $A u$ coated quartz optical flat, and a spherical lens in a distance range from $0.6 \mu \mathrm{m}$ to $6 \mu \mathrm{m}$. The thickness of both metallic layers was $0.5 \mu \mathrm{m}$. The radius of a spherical lens was estimated as $R=11.3 \pm 0.1 \mathrm{~cm}$ (later the improved value of $12.5 \pm 0.3 \mathrm{~cm}$ was published [23]). The measurements were performed at a pressure of $10^{-4}$ Torr at room temperature.

The experimental data were confronted with an ex- pression for the Casimir force between a plate and a sphere made of ideal metals

$$
F_{C}^{(0)}(a)=-\frac{\pi^{3} R}{360} \frac{\hbar c}{a^{3}}
$$

There is the theoretical correction to the Casimir force due to finite conductivity of the metal. In [21] the Casimir force together with the first order conductivity correction was represented in the form

$$
F_{C}^{\left(\delta_{0}\right)}(a)=F_{C}^{(0)}(a)\left(1-4 \frac{\delta_{0}}{a}\right),
$$

where the effective penetration depth of the electromagnetic zero oscillations into the metal is

$$
\delta_{0}=\frac{c}{\omega_{p}}=\frac{\lambda_{p}}{2 \pi}
$$

$\omega_{p}$ and $\lambda_{p}$ are the effective plasma frequency and wavelength of the electrons.

In [21] correction to (2.1) due to nonzero temperature was also considered. Together with this correction the Casimir force is

$$
F_{C}^{(T)}(a)=F_{C}^{(0)}(a)\left[1+\frac{720}{\pi^{2}} f(\xi)\right]
$$

where $\xi=k_{B} T a /(\hbar c), k_{B}$ is the Boltzmann constant, $T$ is the absolute temperature, and $f(\xi)$ is a tabulated function (see, e.g., [24]).

The corrections to the Casimir force (2.1) due to the surface roughness were not considered in [21].

The absolute error of force measurements in [21] was, approximately, $\Delta=10^{-11} \mathrm{~N}$. According to [21, 23] "Agreement with theory at the level of $5 \%$ is obtained". By a theory the formula (2.1) is meant. The statement of [21] is: "data is not of sufficient accuracy to demonstrate the finite temperature corrections". Also data of [21] does not support the presence of the corrections due to finite conductivity of the covering metal.

\section{The experiment by U. Mo- hideen and A. Roy}

In [22] the Atomic Force Microscope was adapted to measure the Casimir force between $A l$ plus $A u / P d$ coated sapphire disk and polystyrene sphere for surface separations between 0.1 to $0.9 \mu \mathrm{m}$. The thicknesses of $A l$ and $A u / P d$ layer were correspondingly $0.3 \mu \mathrm{m}$ and $0.02 \mu \mathrm{m}$. The measurements were performed at a pressure of $5 \cdot 10^{-2}$ Torr at room temperature with a sphere diameter $196 \mu \mathrm{m}$.

The ideal theoretical expression for the Casimir force is given by (2.1) once more. Corrections due to finite conductivity of a metal up to the second order 
in relative penetration depth were accounted for in [22] using the theoretical result of [25]:

$$
F_{C}^{\left(\delta_{0}\right)}(a)=F_{C}^{(0)}(a)\left(1-4 \frac{\delta_{0}}{a}+\frac{72}{5} \frac{\delta_{0}^{2}}{a^{2}}\right) .
$$

The temperature corrections to the Casimir force are given by (2.4). In addition in [22] the roughness of the test bodies was investigated by the Atomic Force Microscope and Scanning Electron Microscope. The average roughness amplitude was estimated to be $A=35 \mathrm{~nm}$. The theoretical expression for the Casimir force with a stochastic roughness correction [25]

$$
F_{C}^{(R)}(a)=F_{C}^{(0)}(a)\left[1+6\left(\frac{A}{a}\right)^{2}\right],
$$

was used in comparison of a theory with experiment.

The absolute error of force measurements in [22] was, approximately, $\Delta=2 \cdot 10^{-12} \mathrm{~N}$. In [22] the root mean square average deviation $\sigma$ ( $\mathrm{rms}$ ) between the experimental and theoretical Casimir force values was chosen as the quantity describing agreement of a theory and experiment. For the Casimir force (2.1) together with the finite conductivity correction (3.1) and roughness correction (3.2) the value $\sigma=1.6 \mathrm{pN}$ was obtained in [22] (temperature corrections are not important in the measurement range under consideration). This deviation is, approximately, $1 \%$ of the force at the smallest surface separations. This value was taken in [22] as a statistical measure of the experimental precision. Note that the ideal Casimir force expression (2.1) along leads to $\sigma=6.3 \mathrm{pN}$ ( $5 \%$ deviation at the smallest separation), (2.1) together with only the finite conductivity correction (3.1) results in a $\sigma=5.5 \mathrm{pN},(2.1)$ together with only the roughness correction (3.2) leads to a $\sigma=48 \mathrm{pN}$ ( $40 \%$ deviation at the closest spacing). What this means is data of [22] support the presence of both corrections due to finite conductivity and due to surface roughness.

\section{Analyzing experimental re- sults}

Let us start with the results of paper [21] which uses a torsion pendulum to measure the Casimir force. As it was already noted in Sec. II experimental data of [21] do not support the presence of finite conductivity correction to the Casimir force. This correction is negative and for the closest spacing $(a=0.6 \mu \mathrm{m})$ can achieve $20 \%$ of $F_{C}^{(0)}$. This was recognized in [21] (see also [26]). The detailed analyses of the finite conductivity correction up to the fourth order in relative penetration depth is contained in [7]. There it was confirmed that the value of the finite conductivity correction to the Casimir force at $a=0.6 \mu \mathrm{m}$ is about $20 \%$ of $F_{C}^{(0)}$ (the attempt to avoid this conclusion, undertaken in [26], fails "due to an invalid manipulation of optical data" [27]). To conclude, the actual value of the finite conductivity correction is in contradiction with the claimed in [21] agreement with the theory at the level of $5 \%$.

Now let us turn to the role of finite temperature and surface roughness corrections in the experiment [21]. According to [23] "...effects of finite temperature and surface roughness, are estimated to be significantly less than $10 \%$..." This statement, however, is incorrect. The roughness correction can achieve $30 \%$ of $F_{C}^{(0)}$ if the large-scale deviations of the boundary surfaces from the perfect shape are present [28]. This is actually the case in the experiment [21] because the lens in use was aspheric [23]. As to the temperature correction it is of $86 \%$ of $F_{C}^{(0)}$ at $a=4 \mu \mathrm{m}, 129 \%$ of $F_{C}^{(0)}$ at $a=5 \mu \mathrm{m}$, and $174 \%$ of $F_{C}^{(0)}$ at $a=6 \mu \mathrm{m}$ at room temperature [7]. But the data of [21] do not support the presence of both roughness and temperature corrections.

¿From the above it becomes clear that the claimed in [21] agreement with the theory at the level of $5 \%$ does not stand up.

We now discuss the main conclusion of the paper [22] in which an atomic force microscope was used to measure the Casimir force. Let us remind that here the experimental data are in agreement with the presence of both roughness and finite conductivity corrections given by Eqs. (3.1) and (3.2). There is one difficulty, however, in the use of Eqs. (3.1) and (3.2). The thing is that they are not exact but perturbative results. For example, the second order term from (3.1) contributes $25 \%$ of $F_{C}^{(0)}$ for Aluminum at the closest spacing $(a=0.12 \mu \mathrm{m})$. Moreover, the second order roughness correction in $(3.2)$ contributes $51 \%$ of $F_{C}^{(0)}$ at $a=0.12 \mu \mathrm{m}$. It is evident that higher order conductivity and roughness corrections should be taken into account to get the theoretical result which would be valid up to $1 \%$ accuracy. That is the reason why the agreement between experimental data and theory at the level of $1 \%$ at the smallest separation claimed in [22] calls for further confirmation.

\section{More complete theory fits experiment}

In this section we present a summary of collaborative experimental and theoretical results obtained in [29]. They make more clear the actual situation with a new measurement of the Casimir force [22] and its agreement with a theory.

In the case that the characteristic lateral sizes of distortions covering the plate and the sphere are small comparing $\sqrt{a R}$ the following general expression for the Casimir force with a roughness correction is valid 
$[28,29]$

$$
\begin{aligned}
& F_{C}^{(R)}(a)=F_{C}^{(0)}(a)\left\{1+6\left[\left\langle f_{1}^{2}\right\rangle\right\rangle\left(\frac{A_{1}}{a}\right)^{2}\right. \\
& \left.-2\left\langle\left\langle f_{1} f_{2}\right\rangle\right\rangle \frac{A_{1}}{a} \frac{A_{2}}{a}+\left\langle\left\langle f_{2}^{2}\right\rangle\right\rangle\left(\frac{A_{2}}{a}\right)^{2}\right] \\
& +10\left[\left\langle\left\langle f_{1}^{3}\right\rangle\right\rangle\left(\frac{A_{1}}{a}\right)^{3}-3\left\langle\left\langle f_{1}^{2} f_{2}\right\rangle\right\rangle\left(\frac{A_{1}}{a}\right)^{2} \frac{A_{2}}{a}\right. \\
& \left.+3\left\langle\left\langle f_{1} f_{2}^{2}\right\rangle\right\rangle \frac{A_{1}}{a}\left(\frac{A_{2}}{a}\right)^{2}-\left\langle\left\langle f_{2}^{3}\right\rangle\right\rangle\left(\frac{A_{2}}{a}\right)^{3}\right] \\
& +15\left[\left\langle f_{1}^{4}\right\rangle\right\rangle\left(\frac{A_{1}}{a}\right)^{4}-4\left\langle\left\langle f_{1}^{3} f_{2}\right\rangle\right\rangle\left(\frac{A_{1}}{a}\right)^{3} \frac{A_{2}}{a} \\
& +6\left\langle\left\langle f_{1}^{2} f_{2}^{2}\right\rangle\right\rangle\left(\frac{A_{1}}{a}\right)^{2}\left(\frac{A_{2}}{a}\right)^{2} \\
& \left.\left.-4\left\langle\left\langle f_{1} f_{2}^{3}\right\rangle\right\rangle \frac{A_{1}}{a}\left(\frac{A_{2}}{a}\right)^{3}+\left\langle\left\langle f_{2}^{4}\right\rangle\right\rangle\left(\frac{A_{2}}{a}\right)^{4}\right]\right\} .
\end{aligned}
$$

Here $f_{1,2}$ are the functions describing surface distortions, $A_{1,2}$ are distortion amplitudes calculated from the middle distortion level. The double angle brackets denote two successive averaging procedures. The first one is the averaging over the surface area of interacting bodies. The second one is over all possible phase shifts between the distortions situated on the surfaces of interacting bodies against each other. This second averaging is necessary because in the experiment [22] the measured Casimir force was averaged over 26 scans of the atomic force microscope.

The roughness of the metal covering was measured with the same atomic force microscope using a standard cantilever having a sharp tip (instead of a sphere). The major distortions are the large separate crystals situated irregularly on the surfaces. They can be modeled approximately by the parallelepipeds of two heights. As the analysis of several AFM images shows, the height of highest distortions is about $h_{1}=40 \mathrm{~nm}$ and of the intermediate ones - about $h_{2}=20 \mathrm{~nm}$. Almost all surface between the distortions is covered by the stochastic roughness of height $h_{0}=10 \mathrm{~nm}$ consisting of small crystals. All together they form the homogeneous background of the averaged height $h_{0} / 2$. The character of roughness on the plate and on the lens is quite similar.

Now it is possible to determine the height $H$ relative to which the middle value of the function, describing the total roughness, is zero. It can be found from the equation

$$
\left(h_{1}-H\right) S_{1}+\left(h_{2}-H\right) S_{2}-\left(H-\frac{h_{0}}{2}\right) S_{0}=0,
$$

where $S_{1,2,0}$ are, correspondingly, the surface areas occupied by distortions of the heights $h_{1}, h_{2}$ and stochastic roughness. Dividing (5.2) into the area of interacting surface $S=S_{1}+S_{2}+S_{0}$ one gets

$$
\left(h_{1}-H\right) v_{1}+\left(h_{2}-H\right) v_{2}-\left(H-\frac{h_{0}}{2}\right) v_{0}=0,
$$

where $v_{1,2,0}=S_{1,2,0} / S$ are the relative parts of the surface occupied by the different kinds of roughness. The analysis of the obtained AFM pictures gives us the values $v_{1}=0.11, v_{2}=0.25, v_{0}=0.64$. Solving Eq. (5.3) we get the height of the zero distortions level $H=12.6 \mathrm{~nm}$. The value of distortion amplitude defined relatively to this level is

$$
A=h_{1}-H=27.4 \mathrm{~nm} .
$$

Below two more parameters will also be used

$$
\begin{aligned}
& \beta_{1}=\frac{h_{2}-H}{A} \approx 0.231, \\
& \beta_{2}=\frac{H-h_{0} / 2}{A} \approx 0.346 .
\end{aligned}
$$

With the help of them the distortion function of the plate can be represented as

$$
f_{1}\left(x_{1}, y_{1}\right)=\left\{\begin{aligned}
1, & \left(x_{1}, y_{1}\right) \in \Sigma_{S_{1}}, \\
\beta_{1}, & \left(x_{1}, y_{1}\right) \in \Sigma_{S_{2}}, \\
-\beta_{2}, & \left(x_{1}, y_{1}\right) \in \Sigma_{S_{0}},
\end{aligned}\right.
$$

where $\Sigma_{S_{1}, S_{2}, S_{0}}$ are the regions of the first interacting body surface occupied by the different kinds of roughness. For a sphere the analogical representation of the distortion function is valid.

Now it is not difficult to calculate the coefficients of expansion (5.1). One example is

$$
\begin{array}{r}
\left\langle\left\langle f_{1} f_{2}\right\rangle\right\rangle=-v_{1}^{2}-2 \beta_{1} v_{1} v_{2}+2 \beta_{2} v_{1} v_{0} \\
\quad-\beta_{1}^{2} v_{2}^{2}+2 \beta_{1} \beta_{2} v_{2} v_{0}-\beta_{2}^{2} v_{0}^{2}=0,
\end{array}
$$

which follows from Eqs. (5.3)-(5.5). The results for the other coefficients are

$$
\begin{aligned}
& \left\langle\left\langle f_{1}^{2}\right\rangle\right\rangle=\left\langle\left\langle f_{2}^{2}\right\rangle\right\rangle=v_{1}+\beta_{1}^{2} v_{2}+\beta_{2}^{2} v_{0}, \\
& \left\langle\left\langle f_{1}^{3}\right\rangle\right\rangle=-\left\langle\left\langle f_{2}^{3}\right\rangle\right\rangle=v_{1}+\beta_{1}^{3} v_{2}-\beta_{2}^{3} v_{0}, \\
& \left\langle\left\langle f_{1} f_{2}^{2}\right\rangle\right\rangle=\left\langle\left\langle f_{1}^{2} f_{2}\right\rangle\right\rangle=0, \\
& \left\langle\left\langle f_{1}^{4}\right\rangle\right\rangle=\left\langle\left\langle f_{2}^{4}\right\rangle\right\rangle=v_{1}+\beta_{1}^{4} v_{2}+\beta_{2}^{4} v_{0}, \\
& \left\langle\left\langle f_{1} f_{2}^{3}\right\rangle\right\rangle=\left\langle\left\langle f_{1}^{3} f_{2}\right\rangle\right\rangle=0, \\
& \left\langle\left\langle f_{1}^{2} f_{2}^{2}\right\rangle\right\rangle=\left(v_{1}+\beta_{1}^{2} v_{2}+\beta_{2}^{2} v_{0}\right)^{2} .
\end{aligned}
$$

Substituting (5.8) into (5.1) we get the final expression for the Casimir force with surface distortions included up to the fourth order in relative distortion amplitude

$$
\begin{gathered}
F_{C}^{(R)}(a)=F_{C}^{(0)}(a)\left\{1+12\left(v_{1}+\beta_{1}^{2} v_{2}\right.\right. \\
\left.+\beta_{2}^{2} v_{0}\right) \frac{A^{2}}{a^{2}}+20\left(v_{1}+\beta_{1}^{3} v_{2}-\beta_{2}^{3} v_{0}\right) \frac{A^{3}}{a^{3}} \\
+30\left[v_{1}+\beta_{1}^{4} v_{2}+\beta_{2}^{4} v_{0}\right. \\
\left.\left.+3\left(v_{1}+\beta_{1}^{2} v_{2}+\beta_{2}^{2} v_{0}\right)^{2}\right] \frac{A^{4}}{a^{4}}\right\} .
\end{gathered}
$$


It should be noted that exactly the same result can be obtained in a very simple way. To do this it is enough to calculate the values of the Casimir force (2.1) for six different distances which are possible between the distorted surfaces, multiply them by the appropriate probabilities and then to summarize the results

$$
\begin{aligned}
F_{C}^{(R)}(a) & =\sum_{i=1}^{6} w_{i} F_{C}^{(0)}\left(a_{i}\right) \equiv v_{1}^{2} F_{0}(a-2 A) \\
+ & 2 v_{1} v_{2} F_{0}\left(a-A\left(1+\beta_{1}\right)\right) \\
& +2 v_{2} v_{0} F_{0}\left(a-A\left(\beta_{1}-\beta_{2}\right)\right) \\
& +v_{0}^{2} F_{0}\left(a+2 A \beta_{2}\right)+v_{2}^{2} F_{0}\left(a-2 A \beta_{1}\right) \\
& +2 v_{1} v_{0} F_{0}\left(a-A\left(1-\beta_{2}\right)\right) .
\end{aligned}
$$

Now let us discuss more in detail the corrections to the Casimir force due to finite conductivity of the covering metals in the experiment [22]. The interacting bodies used in the experiment [22] were coated with $300 \mathrm{~nm}$ of $A l$ in an evaporator. The thickness of this metallic layer is much larger than the penetration depth $\delta_{0}$ of electromagnetic oscillations into $A l$ for the wavelengths (sphere-plate separations) of interest. Taking $\lambda_{p}^{A l}=100 \mathrm{~nm}$ as the approximative value of the effective plasma wavelength of the electrons in $A l$ one gets $\delta_{0}=\lambda_{p}^{A l} /(2 \pi) \approx 16 \mathrm{~nm}$. What this means is the interacting bodies can be considered as made of $A l$ as a whole. Although $A l$ reflects more than $90 \%$ of the incident electromagnetic oscillations in the complete measurement range $100 \mathrm{~nm}<\lambda<950 \mathrm{~nm}$, some corrections to the Casimir force due to the finiteness of its conductivity exist and should be taken into account. In addition, to prevent the oxidation processes, the surface of $A l$ in [22] was covered with $\Delta=20 \mathrm{~nm}$ layer of $60 \% A u / 40 \% \mathrm{Pd}$. The reflectivity properties of this alloy are worse than of $A l$.

We consider firstly the case of small distances $a<$ $500 \mathrm{~nm}$. Here the transmittance of $20 \mathrm{~nm} A u / P d$ films for the characteristic wavelengths contributing to the force value is greater than $90 \%$. This transmission measurement was made by taking the ratio of light transmitted through a glass slide with and without the $A u / P d$ coating in an optical spectrometer. So high transmittance gives the possibility to neglect the $A u / P d$ layers when calculating the Casimir force and to enlarge the distance between the bodies by $2 \Delta=40 \mathrm{~nm}$ when comparing the theoretical and experimental results.

For pure $A l$ the Casimir force with finite conductivity corrections up to the 4 th order in relative penetration depth can be obtained from the interpolation formula [8]

$$
\begin{gathered}
F_{C}^{\left(\delta_{0}\right)}(a+2 \Delta)=F_{C}^{(0)}(a+2 \Delta)\left[1-4 \frac{\delta_{0}}{a+2 \Delta}\right. \\
+\frac{72}{5} \frac{\delta_{0}^{2}}{(a+2 \Delta)^{2}}-\frac{152}{3} \frac{\delta_{0}^{3}}{(a+2 \Delta)^{3}}
\end{gathered}
$$

$$
\left.+\frac{532}{3} \frac{\delta_{0}^{4}}{(a+2 \Delta)^{4}}\right] .
$$

Now we combine both corrections - one due to the surface roughness and the second due to the finite conductivity of the metal. For this purpose we substitute the quantity $F_{C}^{\left(\delta_{0}\right)}\left(a_{i}\right)$ from (5.11) into Eq. (5.10) instead of $F_{C}^{(0)}\left(a_{i}\right)$. The result is

$$
F_{C}(a)=\sum_{i=1}^{6} w_{i} F_{C}^{\left(\delta_{0}\right)}\left(a_{i}\right)
$$

In the range of large distances $600 \mathrm{~nm}<a<900 \mathrm{~nm}$ there is no necessity to take into account any corrections to the Casimir force due to the large scatter in experimental points due to the experimental uncertainty. Here the ideal expression (2.1) for the Casimir force can be used (see [29] for the details). It gives the rms deviation between theory and experiment of $1.5 \mathrm{pN}$.

Now we compare the experimental and theoretical results in the range of smaller values of the distance $80 \mathrm{~nm} \leq a \leq 460 \mathrm{~nm}$ (or, between $A l, 120 \mathrm{~nm} \leq a+2 \Delta \leq$ $500 \mathrm{~nm})$. Here the Eq. (5.12) should be used for the Casimir force. In Fig. 1 the Casimir force $F_{C}^{(0)}(a+2 \Delta)$ from (2.1) is shown by the dashed curve. The solid curve represents the dependence calculated according to Eq. (5.12). The open squares are the experimental points [29].

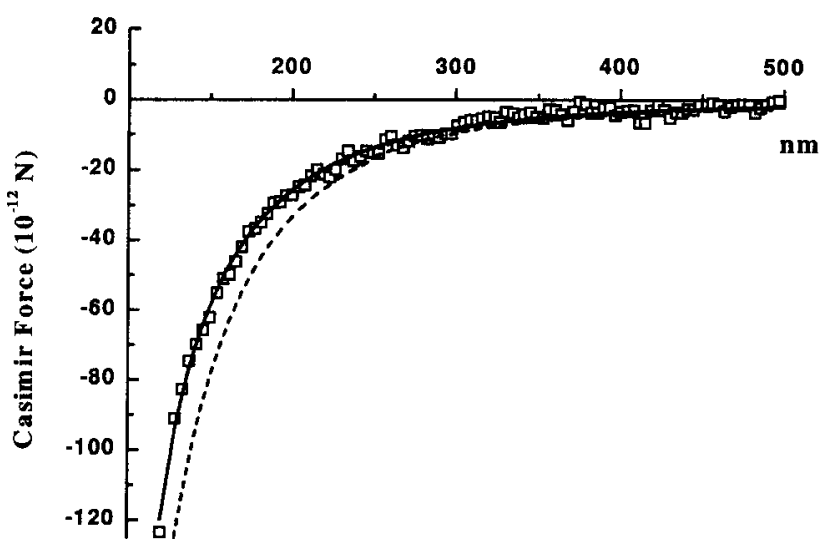

Figure 1. The measured average Casimir force for small distances as a function of plate-sphere separation is shown as open squares. The theoretical Casimir force with corrections to surface roughness and finite conductivity is shown by the solid line, and without any correction by the dashed line.

Taking into account all one hundred experimental points belonging to the range of smaller distances we get for the solid curve the value of the root mean square deviation between theory and experiment $\sigma_{100}=1.5 \mathrm{pN}$. If we consider more narrow distance interval $80 \mathrm{~nm} \leq$ $a \leq 200 \mathrm{~nm}$ which contains thirty experimental points it turns out that $\sigma_{30}=1.6 \mathrm{pN}$ for the solid curve. In all the measurement range $80 \mathrm{~nm} \leq a \leq 910 \mathrm{~nm}$ the root 
mean square deviation is $\sigma_{223}=1.4 \mathrm{pN}$ (223 experimental points). What this means is that the dependence (5.12) gives equally good agreement with experimental data in the region of small distances (for the smallest ones the relative error of force measurement is about $1 \%$ ), in the region of large distances (where it gives the same result as (2.1) because the relative error is rather large) and in the whole measurement range. If one uses less sophisticated expressions for the corrections to the Casimir force due to the surface roughness and finite conductivity, the value of $\sigma$ calculated for small $a$ would be larger than in the whole range [22].

It is interesting to compare the obtained results with those given by Eq. (2.1), i.e. without account of any correction. In this case for the interval $80 \mathrm{~nm} \leq$ $a \leq 460 \mathrm{~nm}$ (one hundred experimental points) we have $\sigma_{100}^{0}=8.7 \mathrm{pN}$. For the whole measurement range $80 \mathrm{~nm} \leq a \leq 910 \mathrm{~nm}$ (223 points) there is $\sigma_{223}^{0}=5.9 \mathrm{pN}$. It is evident that without appropriate treatment of the corrections to the Casimir force the value of the root mean square deviation is not only larger but also depends significantly on the measurement range.

The comparative role of each correction is also quite obvious. If we take into account only roughness correction according to Eq.(5.10), then one obtains for the root mean square deviation in different intervals: $\sigma_{30}^{R}=22.8 \mathrm{pN}, \sigma_{100}^{R}=12.7 \mathrm{pN}$ and $\sigma_{223}^{R}=8.5 \mathrm{pN}$. At $a+2 \Delta=120 \mathrm{~nm}$ the correction is $17 \%$ of $F_{C}^{(0)}$. For the single finite conductivity correction calculated by Eq. (5.11) it follows: $\sigma_{30}^{\delta}=5.2 \mathrm{pN}, \sigma_{100}^{\delta}=3.1 \mathrm{pN}$ and $\sigma_{223}^{\delta}=2.3 \mathrm{pN}$. At $120 \mathrm{~nm}$ this correction contributes $-34 \%$ of $F_{C}^{(0)}$. (Note, that the contribution of both corrections is $-22 \%$ of $F_{C}^{(0)}$ at $120 \mathrm{~nm}$, so that their non-additivity is demonstrated most clearly.)

\section{Conclusions and discussion}

In the above, it was shown that the experimental results of [22] are in excellent agreement with the more exact theory of the Casimir force taking into account corrections up to the 4 th order both in surface roughness and finite conductivity. The $1 \%$ agreement between a theory and experiment was confirmed at the smallest separations, i.e. the same as was claimed originally in [22]. There are two reasons why one and the same set of data is in approximately the same agreement with two theories of different accuracy. Firstly, the higher order corrections for the surface roughness and finite conductivity have different signs (positive and negative, respectively) and partly compensate each other. Secondly, for large separations the relative error of force measurements is so large that both theoretical approaches are equivalent. The account of the region of large separations leads to almost equivalent rms deviations in all measurement range.

At the same time, there exists an important differ- ence between two theoretical approaches in their relation to data. With the less accurate theoretical approach used in [22], the value of rms deviation depends significantly on the distance range. With the more exact approach of [29], the value of $\sigma$ is almost the same for small separations, large separations and in all measurement range. This denounces doubts raised in [26] (see also S.K. Lamoreaux comment [31] and U. Mohideen and A. Roy reply [32]): experimental data of [22] agree with a theory at a level of $1 \%$ at the smallest separations.

It is notable also that the method of atomic force microscopy shows a considerable promise in the measurement of the Casimir force (the results of new experiment with lower roughness and systematic errors have been published recently [33]). There is reason to hope that during the next few years the different aspects of the Casimir effect will be examined experimentally in more detail.

\section{Acknowledgment}

The author is grateful to the Organizing Committee of the XX ENFPC for their invitation to present this talk and for kind hospitality at São Lourenço during the Conference.

\section{References}

[1] H. B. G. Casimir, Proc. Kon. Nederl. Akad. Wet. 51, 793 (1948).

[2] V. M. Mostepanenko and N. N. Trunov, The Casimir Effect and Its Applications (Clarendon Press, Oxford, 1997).

[3] I. Brevik and H. Kolbenstvedt, Ann. Phys. 143, 179 (1982).

[4] K. A. Milton, Ann. Phys. 150, 432 (1983).

[5] P. Candelas and S. Weinberg, Nucl. Phys. B 237, 397 (1984).

[6] G. L. Klimchitskaya, E. R. Bezerra de Mello, and V. M. Mostepanenko, Phys. Lett. A 236, 280 (1997).

[7] M. Bordag, B. Geyer, G. L. Klimchitskaya, and V. M. Mostepanenko, Phys. Rev. D 58, 075003 (1998).

[8] M. Bordag, B. Geyer, G. L. Klimchitskaya, and V. M. Mostepanenko, Phys. Rev. D 60, 055004 (1999).

[9] L. H. Ford, Phys. Rev. D 21, 933 (1980).

[10] Ya. B. Zel'dovich and A. A. Starobinsky, Sov. Astron. Lett. (USA) 10, 323 (1984).

[11] A. Vilenkin and E. P. S. Shellard, Cosmic Strings and Other Topological Defects (Cambridge University Press, Cambridge, 1994).

[12] P. W. Milonni, The Quantum Vacuum (Academic Press, San Diego, 1994).

[13] G. Plunien, B. Müller, and W. Greiner, Phys. Rep. C 134, 87 (1986). 
[14] T. H. Boyer, Phys. Rev. 174, 1764 (1968).

[15] M. Krech and S. Dietrich, Phys. Rev. A 46, 1886 (1992).

[16] E. J. Kelsey and L. Spruch, Phys. Rev. A 18, 15 (1978).

[17] G. Barton, Proc. Roy. Soc. Lond. A 410, 141 (1987).

[18] S. K. Blau, M. Visser, and A. Wipf, Nucl. Phys. B 310, 163 (1988).

[19] M. J. Sparnaay, Physica 24, 751 (1958).

[20] P. H. G. M. van Blokland and J.T.G. Overbeek, J. Chem. Soc. Faraday Trans. 74, 2637 (1978).

[21] S. K. Lamoreaux, Phys. Rev. Lett. 78, 5 (1997).

[22] U. Mohideen and A. Roy, Phys. Rev. Lett. 81, 4549 (1998).

[23] S. K. Lamoreaux, Phys. Rev. Lett. 81, 5475 (1998), Erratum.

[24] L. S. Brown and G. J. Maclay, Phys. Rev. 184, 1272 (1969).
[25] V. B. Bezerra, G. L. Klimchitskaya, and C. Romero, Mod. Phys. Lett. A 12, 2613 (1997).

[26] S. K. Lamoreaux, Phys. Rev. A 59, R3149 (1999).

[27] A. Lambrecht and S. Reynaud, e-print quantph/9907105.

[28] G. L. Klimchitskaya and Yu. V. Pavlov, Int. J. Mod. Phys. A 11, 3723 (1996).

[29] G. L. Klimchitskaya, A. Roy, U. Mohideen, and V. M. Mostepanenko, Phys. Rev. A 60, 3487 (1999).

[30] M. Bordag, G. L. Klimchitskaya, and V. M. Mostepanenko, Int. J. Mod. Phys. A 10, 2661 (1995).

[31] S. K. Lamoreaux, Phys. Rev. Lett. 83, 3340 (1999).

[32] U. Mohideen and A. Roy, Phys. Rev. Lett. 83, 3341 (1999).

[33] A. Roy, C. Y. Lin, and U. Mohideen, Phys. Rev. D 60, 111101 (1999). 